\title{
АВТОМАТИЗИРОВАННЫЙ АЛГОРИТМ ФОРМИРОВАНИЯ НАПРАВЛЕННОГО ИНФОРМАЦИОННОГО ПОТОКА В ЦИФРОВОЙ СРЕДЕ (НА ПРИМЕРЕ ТЕХНОЛОГИЙ ВИЭ)
}

\author{
(C) 2021 Конников Евгений Александрович \\ кандидат экономических наук, доцент, Высшая инженерно-экономическая школа \\ Санкт-Петербургский политехнический университет Петра Великого, Россия, Санкт-Петербург \\ E-mail: konnikov.evgeniy@gmail.com \\ (c) 2021 Вольвач Олеся Сергеевна \\ Высшая инженерно-экономическая школа \\ Санкт-Петербургский политехнический университет Петра Великого, Россия, Санкт-Петербург \\ E-mail:volvach_o@mail.ru \\ (c) 2021 Конникова Ольга Анатольевна \\ кандидат экономических наук, доцент, кафедра маркетинга \\ Санкт-Петербургский государственный экономический университет (СПбГЭУ), \\ Россия, Санкт-Петербург \\ E-mail: olga.a.konnikova@gmail.com
}

Процесс перехода к цифровой экономике в первую очередь характеризуется содержательной трансформацией процесса потребления. Ключевым свойством данной трансформации в первую очередь является транспонирование коммуникационного процесса в цифровую среду. Таким образом, управление процессом потребления на данный момент возможно в первую очередь за счет управления информационным полем потребителя. Особую значимость данный процесс приобретает для инновационных категорий товаров, изначально не входящих в информационное поле потенциального потребителя. Одним из значимых примеров подобных товаров являются технологии возобновляемых источников энергии. В рамках статьи [1] ранее был разработан маркетинговый инструмент, направленный на последовательное изменение восприятия возобновляемых источников энергии потенциальным потребителем, реализуемый в цифровой среде как наиболее доступной и легкой для восприятия современным потребителем. В рамках данной статьи правится математическая детализация данного алгоритма и его автоматизация посредствам языка программирования Python.

Ключевые слова: возобновляемые источники энергии, окно Овертона, цифровая среда, направленный информационный поток.

Разработанный ранее и представленный в статье [1] алгоритм последовательного изменения восприятия ВИЭ потенциальным потребителем в первую очередь требует математической детализации. Каждый из выделенных в рамках алгоритма этапов предполагает формирование количественно специфицированных массивов информации, описывающих потенциальных потребителей. В рамках первичного этапа осуществляется поиск и идентификация потенциальных потребителей. Данный этап является наиболее вариативным с точки зрения подхода к формированию конечного результата. Данный тезис обусловлен в первую очередь многомерностью возможностей использования технологий генерации энергии на основе ВИЭ. Потенциальных потребителей ВИЭ можно в первую очередь дифференцировать на совокупность юридических лиц и совокупность домохозяйств. При этом, целеполагание субъектов, представляющих каждую из совокупностей, может принципиально отличаться. В частности, для юридических лиц можно выделить следующую совокупность ценностей, формируемых использованием ВИЭ:

- Сравнительно низкая себестоимость генерируемой энергии. Данный пункт является наиболее значимым, однако наименее достижимым 
на данном технологическом этапе развития. Достижение сравнительно низкой себестоимости генерируемой энергии как правило возможно в условиях отсутствия централизованного энергоснабжения, а также сравнительно высокой стоимости (или принципиальной недоступности) ископаемого топлива, такого как природный газ, нефть или уголь.

- Сравнительно высокий уровень энергетической независимости/автономности. Относительная независимость от централизованных генерирующих мощностей позволит предприятию быть значительно более энергетически устойчивым в условиях политической, социальной или природной нестабильности, спровоцированной революциями, восстаниями или природными катастрофами.

- Маркетинговый эффект, вызванный ассоциативной спецификой. На данный момент тематика устойчивого развития значимо развивается и захватывает ума множества потенциальных потребителей ТРУ. Одним из ключевых аспектов концепции устойчивого развития является экологическая устойчивость. Использование ВИЭ позволит сформировать маркетинговый капитал, за счет позиционирования предприятия как экологически устойчивого, что в свою очередь позволит привлечь дополнительных потребителей.

Данная совокупность ценностей может быть экстраполирована и на домохозяйства. Следовательно, процесс определения потенциальных потребителей в первую очередь должен основываться на совокупности приведенных ценностей. Результатом реализации данного этапа является массив цифровых социальных профилей потенциальных потребителей. Данные профили могут быть определены на основе существующей совокупности социальных медиа, таких как Instagram, Facebook, twitter и ВКонтакте. Каждая из представленных социальных сетей обладает уникальной спецификой, в первую очередь с точки зрения превалирующего способа представления контента, а следовательно, целеполагания посещения. В рамках РФ наиболее распространенной и универсальной с точки зрения способа представления контента является социальная сеть ВКонтакте. Подавляющая часть потребляемого в рамках данной социальной сети контента консолидируется в рамках единого, специфицированного в зависимости от предпочтений пользователя, информационного потока. Данный информационный поток сформирован на основе как текстовой информации, так и массива аудиовизуальных единиц, что позволяет готовить о смешанной форме потребляемого пользователями контента. Данный контент является непосредственно аналитическим базисом сформированного алгоритма. Обработка массива аудиовизуальных единиц в данном случае не является целесообразным, так как обучение нейронной сети (или иного инструмента, позволяющего распознавать аудиовизуальные образы) требует тематической конкретизации, что в свою очередь не представляется возможным, так как потенциальные потребители технологий генерации энергии на основе ВИЭ являются крайне дифференцированными с точки зрения комплекса интересов. Таким образом, аналитический базис разработанного алгоритма может быть ограничен исключительно массивами текстовой информации.

Процесс обработки массивов текстовой информации, представленной в естественной форме, предполагает выделение тех или иных содержательных характеристик контента и их сравнительную квантификацию. Данный процесс предполагает первичное определение элементарных содержательных единиц текстовой информации, которыми выступают токены. Токены - это лексемы, обладающие содержательной значимостью и приведенное к базовой словарной форме. Процесс выделения массива токенов на основе массива текстовой информации называется токенизацией. Значимость (удельный вес) каждого из выделенных токенов может быть определено частотой упоминания в рамках сформированного массива. Сформированный в результате массив токенов в полной мере описывает информационную среду потенциального потребителя, и может быть дифференцирован в соответствии с содержательнотематической спецификой.

Для целей оценки присутствия тематики ВИЭ в первую очередь необходимо также представить данную содержательно-тематическую компоненту массивом токенов. Следовательно, параллельно токенизации массивов текстовой информации, описывающей информационную среду потенциальных потребителей, необходимо токенизировать массив текстовой информации, описывающей актуальную тематику ВИЭ. 
Оценить степень представленности тематики ВИЭ в информационном поле потенциального потребителя можно посредствам следующей формулы:

$$
K_{\text {r.e.s. }}^{i}=\frac{m\left(T_{i} \cap T_{\text {r.e.s.s }}\right)}{m\left(T_{\text {r.e.s. }}\right)}
$$

Где:

$K_{r . e . s .}^{i}-$ коэффициент присутствия тематики ВИЭ в информационном поле потенциального потребителя і.

$T_{i}$ - множество токенов, описывающих информационное поле потенциального потребителя i.

$T_{\text {r.e.s. }}-$ множество токенов, описывающих актуальную тематику ВИЭ.

$m\left(T_{i} \cap T_{\text {r.e.s. }}\right)-$ количество общих токенов для множества, описывающего информационное поле потенциального потребителя і и актуальную тематику ВИЭ.

$m\left(T_{\text {r.e.s. }}\right)$ - количество токенов в множестве, описывающем актуальную тематику ВИЭ.

Рассчитываемый коэффициент является исключительно сравнительным и не может интерпретироваться напрямую. В первую очередь данный коэффициент необходимо для определения баланса направленного цифрового информационного потока, позволяющего постепенно интегрировать ВИЭ в поле интересов потенциального потребителя. Информационное поле потенциального потребителя, в рамках сформированного алгоритма, может быть дифференцировано на направленный цифровой информационной поток, посвященный ВИЭ, и текущий информационный поток, формируемый информационной средой потенциального потребителя. В свою очередь направленный цифровой информационной поток может быть дифференцирован на поток, содержащий информацию исключительно о ВИЭ и поток, содержащий информацию о ВИЭ в контексте интересов потенциальных потребителей. Баланс приведенных трех потоков определяется непосредственно рассчитанным ранее коэффициентом присутствия тематики ВИЭ в информационном поле потенциального потребителя. Логика распределения удельного веса между потоками определяется функцией последовательного наращивания тематики ВИЭ. Так как предлагаемый алгоритм основан на принципах «Окна Овертона», первичным является поток, содержащий информацию о ВИЭ в контексте интересов потенциальных потребите- лей. При этом его удельный вес не должен превышать условно допустимого уровня. Данный тезис обусловлен необходимостью возможного ограничения осмысления процесс интеграции со стороны потенциального потребителя. Авторы данного исследования предполагают, что условно эффективной пропорцией распределения потока, содержащего информацию о ВИЭ в контексте интересов потенциальных потребителей, является один к десяти. Следовательно, если коэффициент присутствия тематики ВИЭ в информационном поле потенциального потребителя составит 0,5\%, эффективной долей потока, содержащего информацию о ВИЭ в контексте интересов потенциальных потребителе, будет составлять 5\%. Данная пропорция позволит сделать данный информационный поток идентифицируемым в рамках общего информационного потока, и при этом не вызовет подозрения со стороны потенциального потребителя. При этом, поток, содержащий информацию исключительно о ВИЭ, является значительно более значимым, с точки зрения распространения целевой информации, однако при этом он с большей долей вероятности не вызовет интереса у потенциального потребителя. Таким образом, можно предположить, что эффективная пропорция в данном случае будет составлять один к одному. Следовательно, если коэффициент присутствия тематики ВИЭ в информационном поле потенциального потребителя составит 0,5\%, эффективной долей потока, содержащего информацию исключительно о ВИЭ, будет составлять 0,5\%. Базой для расчета могут выступать совокупность потенциальных единиц демонстрируемого контента или среднее число единиц контента, просматриваемых потенциальным потребителем за анализируемый период времени. Безусловно вторая метрика является значительно более эффективной, однако далеко не каждая социальная сеть позволяет определить данную величину, в то время как совокупность потенциальных единиц демонстрируемого контента может быть идентифицировано на основе парсинга актуальной информации, сгенерированной в рамках информационных ресурсов, на которые подписан потенциальный пользователь. Однако, данный параметр необходимо скорректировать в соответствии со среднем временем, которое потенциальны пользователь проводит в социальной сети. Таким образом, долевое соотношение компонент цифрового потока может быть опре- 
делено следующим образом:

$$
\begin{aligned}
& d_{r . e . s .1}^{i}=K_{r . e . s .}^{i} \times 10 \times k_{o n}^{i} \times N^{i} \\
& d_{\text {r.e.s. } 2}^{i}=K_{\text {r.e.s. }}^{i} \times k_{o n}^{i} \times N^{i} \\
& d_{\text {c.i. }}^{i}=k_{o n}^{i} \times\left(N^{i}-d_{\text {r.e.s. } 1}^{i}-d_{\text {r.e.s. } 2}^{i}\right)
\end{aligned}
$$

Где:

$d_{r . e . s .1}^{i}-$ количество единиц информационного потока, содержащего информацию о ВИЭ в контексте интересов потенциального потребителя і.

$k_{o n}^{i}-$ коэффициент присутствия потенциального потребителя і в социальной сети.

$N^{i}$ - совокупность потенциальных единиц демонстрируемого потенциальному потребителю і контента.

$d_{r . e . s .2}^{i}-$ количество единиц информационного потока, содержащего информацию исключительно о ВИЭ.

$d_{c . i .}^{i}$ - количество единиц информационного потока, содержащего информацию исключительно в поле интересов потенциального потребителя i.

На основе рассчитанных количественных параметров, в первую очередь определяется количество единиц контента, посвященного ВИЭ. Подбор данного контента производится экспертно, или может быть частично автоматизирован. На основе сформированного ранее множества токенов, описывающих информационное поле потенциального потребителя, может быть определена группа тематик, первично интересующих потенциального потребителя. Информационные единицы, одновременно принадлежащие к какой-либо из выявленных тематик и тематике ВИЭ, могут формировать первичный массив направляемых в информационное поле потенциального потребителя единиц. На наш взгляд, в данном случае целесообразно использовать смешанный подход, предполагающий первичную автоматизированную выгрузку массива информационных единиц, описывающих новостную повестку, связанную с ВИЭ, а также коррелирующих с областью интересов потенциального потребителя. Далее предполагается экспертный отбор наиболее значимых и подходящих информационных единиц, для формирования направленного информационного потока.

Сформированный массив информационных единиц, посвященных ВИЭ, необходимо напра- вить в информационное поле потенциального потребителя. Данный процесс предполагает следующие возможные инструменты:

1. Прямое необезличенное направление информационных единиц, посвященных ВИЭ, в информационное поле потенциального потребителя через существующие каналы связи. Как правило социальные сети предполагают два возможных необезличенных канала связи - личные сообщения и публичные обращения. В частности, социальная сеть ВКонтакте предполагает оба. Использование данного инструмента, с одной стороны, является наименее эффективным, так как в сознании потребителя данный информационный поток может восприниматься как условная реклама и неосознанно отфильтровываться сознание. В тоже время данный инструмент является наиболее доступным и наименее вариативным с точки зрения достижения направленным информационным потоком потенциального потребителя. Таким образом, в том случае, если потенциальный потребитель некритически относится к подобного рода обращению, данный инструмент может быть эффективен.

2. Прямое эффективно-аффилированное направление информационных единиц, посвященных ВИЭ, в информационное поле потенциального потребителя через существующие каналы связи. Данный инструмент предполагает возможность обращение к потенциальному потребителя от имени авторитетного/известного/близкого ему человека или организации/сообщества. Использование данного инструмента крайне ограничено, в первую очередь из-за сложности формирования комплексных социальных связей. Безусловно, данный метод является наиболее эффективным, за счет использования изначально сформированного коммуникативного канала между потенциальным потребителем и обращающимся к нему субъектом. Таким образом, при наличии возможности использования данного инструмента, его применение наиболее целесообразно.

3. Интеграция направленного информационного потока, посвященного ВИЭ, в информационный поток, генерируемый источниками базового для потенциального потребителя информационного потока. Механика реализации данного инструмента предполагает коммуникацию с представителями информационного поля потенциального потребителя (как физическими лицами, так и пабликами) с целью размещения 
в их публичном поле информационных единиц направленного информационного потока, посвященного ВИЭ, сформированного для потенциального потребителя. Данный инструмент является наиболее вариативным, с точки зрения достижения сформированным контентом потенциального потребителя, так ка алгоритмы социальных сетей как правило формирует информационный поток для пользователя на основе анализа его предпочтений, что приводит к дифференциации всего существующего в информационном поле пользователя контента с точки зрения удельного веса. Таким образом, использование данного инструмента целесообразно исключительно при относительной массовости, достигая тем самым эффекта отдачи от масштаба. Повсеместное применение данного инструмента также ограничивается необходимостью формирования комплексных социальных связей с представителями информационного поля потенциального потребителя, что в целом роднит данный инструмент со вторым инструментом.

4. Интеграция направленного информационного потока, посвященного ВИЭ, в информационное поле потенциального потребителя в качестве таргетированной рекламы. Таргетированная реклама - это направленное воздействие на целевую аудиторию, преподносимая как естественная часть информационного потока, вследствие чего как правило воспринимаемая сознанием потенциального потребителя не как реклама. Подавляющее большинство социальных сетей поддерживает возможность организации и настройки таргетированной рекламы. Однако, таргетированная реклама не может быть настроена на уникального пользователя, исключительно на совокупность пользователей, соответствующих определенным характеристикам. При этом разные социальные сети предоставляют разные возможности настройки, что определяет необходимость привлечения дополнительного специалиста (таргетолога), компетентного в данном вопросе. Для целей сформированного алгоритма данный инструмент является наиболее сбалансированным, так как он предполагает значимую вероятность достижения сформированным информационным потоком, посвященным ВИЭ, потенциального потребителя и не требует формирования комплексных социальных контактов. С экономической точки зрения данный инструмент безусловно затратоёмкий, так как каждое выводимое таргетированное сообщение оплачивается отдельно, однако, данный затраты являются прогнозируемыми.

Безусловно приведенные инструменты могут использоваться параллельно и балансироваться относительно специфики потенциального потребителя и возможностей выгодоприобретателя. Однако четвертый инструмент - таргетированная реклама - является наиболее универсальным и целесообразным к использованию.

По результатам интеграции сформированного информационного потока, посвященного ВИЭ, в информационное поле потенциального потребителя необходим анализ динамики коэффициента присутствия тематики ВИЭ в информационном поле потенциального потребителя. Так как данный коэффициент определяется не на основе фактического информационного потока, направленного на потенциального потребителя в рамках той или иной социальной сети, а определяется исключительно на основе квантификации потенциального информационного потока, можно предположить, что потенциальный потребитель при проявлении интереса к контенту, посвященному ВИЭ, будет интегрировать данную тематику в свое информационное поле (вступать в соответствующие сообщества, изыскивать информацию относительно ВИЭ, а также просто проводить больше времени в прочтении продемонстрированной таргетированной рекламы, посвященной ВИЭ), что в свою очередь обязательно будет учтено алгоритмами социальной сети. Таким образом, в информационном поле потенциального потребителя тематика ВИЭ может начать приобретать больший удельный вес, что безусловно скажется на рассчитываемом коэффициента. Увеличение данного коэффициента неизбежно приведет к наращиванию направленного информационного потока, посвященного ВИЭ, в общем информационном потоке потенциального потребителя. Результатом описанного процесса неизбежно станет проникновение тематики ВИЭ в область пассивных (как минимум) интересов потенциального потребителя, или как минимум данная тематика станет для потенциального потребителя обыденным, что в свою очередь приведет к осмыслению потенциальным потребителем возможных решений поставленных перед ним энергетических задач и использованием технологических решений, основанных на ВИЭ. 
Представленные инструменты подразумевают необходимость извлечения из цифровой среды, агрегирование, токенизацию и квантификацию значительных массивов естественной цифровой информации, описывающей информационное поле потенциального потребителя. Данный процесс может быть в виде универсального трехэтапного алгоритма. Визуализация данного алгоритма представлена на рисунке 1.

Как можно видеть, первый этап подразумевает формирование двух массивов информации - информации, опивающей информационное поле потенциального потребителя и информации, опивающей информационное поле тематики ВИЭ. Источниками данной информации могут быть дифференцированы, однако перечным источником выступают социальные сети потенциального потребителя.

В рамках данного исследования существует объективное ограничение, обусловленное относительной сложностью и высокой стоимостью парсинга таких социальных сетей как Factbook или Twitter, вследствие чего далее рассматривается социальная сеть ВКонтакте. Следует акцентировать внимание на том, что данный пример является исключительно локальным и призван с первую очередь описать представленный алгоритм. На рисунке 2 представлен Программный алгоритм извлечения текстовой информации, формирующей информационное поле потенциального потребителя.

Представленный алгоритм основан на использование официального АРI ВКонтакте. Результатом применения данного алгоритма является структурированный массив текстовой информации, который для целей дальнейшей квантификации в первую очередь необходимо токенизировать. Данный процесс подразумевает в первую очередь первичное разделение массива текстовой информации на элементарные лексемы (токены), лемматизация выделенных токенов с предварительным определением соответствия частей речи и исключение низкосодержательных токенов. На рисунке 3 представлен программный алгоритм токенизации текстовой информации, формирующей информационное поле потенциального потребителя.

Как можно видеть, основой данного алгоритма выступают инструменты библиотеки nltk. Для целей описания информационного поля актуальной тематики ВИЭ используются идентичные алгоритмы. В завершении производится сопоставление сформированных множеств, результатом чего является коэффициент присутствия тематики ВИЭ в информационном поле потенциального потребителя.
Извлечение информации, опивающей информационное поле потенциального потребителя

2.

3. потенциального потребителя
Извлечение информации, опивающей информационное поле тематики ВИЭ

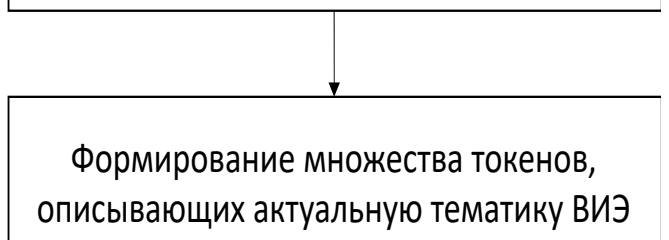


1) Установка необходимых инструментальных библиотек:

- requests - библиотека инструментов извлечения информации из сети.

- datetime - библиотека инструментов трансформации временных данных

$\triangle\{$ from requests import get

from datetime import datetime, timedelta

2) Определяем входные параметры извлекаемой информации:

- user_id-ID анализируемого пользователя.

- content_date_limit - пограничная дата размещения извлекаемого контента.

- $\quad$ params - технические параметры запроса к API

(user_id = ID анализируемого пользователя content_date_limit $=$ datetime.today () - timedelta(days $=7)$ params $=\{$ 'access_token': ключь доступа API, 'v':'5.130', 'user_id':user_id, 'count':1000\}

3) Формирование массива ID контент генераторов:

- $\quad$ url-метод обращения к API.

- groups_0 - изначальный массив информации о группах пользователя.

- groups_ids - массив ID групп пользователя.

- friends_0-изначальный массив информации о друзьях пользователя

- friends_ids - массив ID друзей пользователя.

- inf_ids - совокупный массив ID контент генераторов.

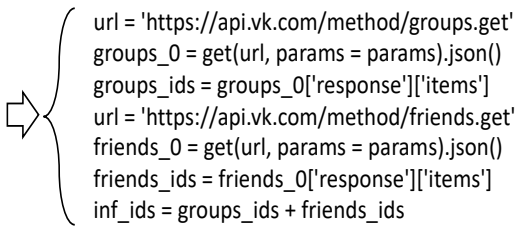

content_texts $=[]$

for id_num in inf_ids:

url = 'https://api.vk.com/method/wall.get'

params $=\{$ 'access_token': ключь доступа API, 'v':'5.130', 'user id':id num, 'count':10\}

content $=$ get (url, params $=$ params).json ()

if list(content.keys())[0] == 'response':

for component in content['response']['items']

if datetime.fromtimestamp(component['date']) <= content_date_limit:

if len(component['text']) $>10$

content_texts.append(component['text'])

Рисунок 2. Программный алгоритм извлечения текстовой информации, формирующей информационное поле потенциального потребителя

1) Установка необходимых инструментальных библиотек:

- word tokenize - инструмент токенизации текстового массива.

pos_tag - инструмент определения части речи токена.

- WordNetLemmatizer - инструмент лемматизации текстового массива.

stopwords - совокупность низкосодержательных токенов.

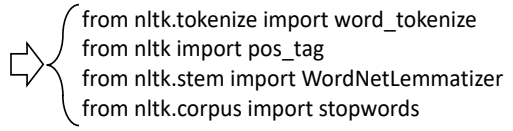

2) Формирование массивов входных параметров:

- $\quad$ stop words - массив низкосодержательных токенов.

noun - список тегов, определяющих существительные. verb - список тегов, определяющих глаголы.

adj - список тегов. определяющих прилагательные.

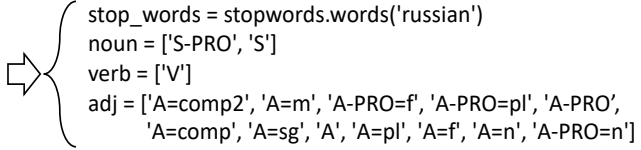

3) Формирование массива токенов, описывающих информационную среду анализируемого пользователя:

tokens final - массив токенов, описывающих информационную среду анализируемого пользователя.
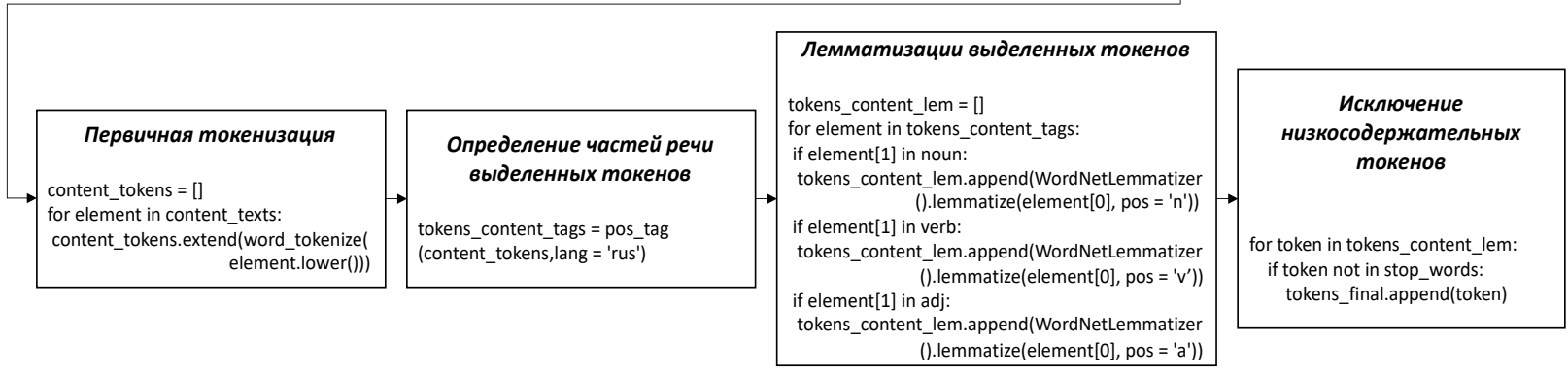

Рисунок 3. Программный алгоритм токенизации текстовой информации, формирующей информационное поле потенциального потребителя 


\section{Библиографический список}

1. Конников Е.А., Вольвач О.С., Конникова О.А. Маркетинговые решений в сфере альтернативной энергетики, основанные на формировании направленного информационного потока в цифровой среде // Экономические науки. 2020. № 193. С. 63-68.

2. Велькин В.И., Щелоков Я.М. Возобновляемая энергетика и энергосбережение. Издательство Уральского университета, 2020.

3. Городов Р.В., Губин В.Е., Матвеев А. С. Нетрадиционные и возобновляемые источники энергии. Издательство Томского политехнического университета, 2009.

4. Конникова О.А., Конников Е.А. Синтаксический анализ поведения потребителей в цифровой среде. В сб. Маркетинг сотворчества и глобальные коммуникации доверия. СПб.: Изд-во СПбГЭУ, 2020. С. $132-145$.

5. Родионов Д.Г., Конников Е.А., Куракин В.И. Оценка маркетинговой надежности инновационных решений (на примере нефтепромыслового оборудования) // Экономические науки. 2020. № 193. С. 388-393.

6. Родионов Д.Г., Конников Е. А., Мугутдинов Р. М. Системный анализ конкурентоспособности цифрового предприятия в рамках информационной среды // Экономические науки. 2020. № 193. С. 394-401.

7. Родионов Д.Г., Конников Е.А., Конникова О.А. Методология системного анализа информационной среды // Экономические науки. 2021. № 196. С. 160-174. 